PROCEEDINGS OF THE

AMERICAN MATHEMATICAL SOCIETY

Volume 132, Number 1, Pages 107-115

S 0002-9939(03)07146-6

Article electronically published on July 14, 2003

\title{
ALMOST CONSTRAINED SUBSPACES OF BANACH SPACES
}

\author{
PRADIPTA BANDYOPADHYAY AND S. DUTTA
}

(Communicated by Jonathan M. Borwein)

\begin{abstract}
In this paper, we obtain some sufficient conditions for an almost constrained subspace to be constrained (in fact, by a unique norm 1 projection), which improves significantly upon all existing conditions of similar type with significantly simpler proofs.
\end{abstract}

\section{INTRODUCTION}

Let $X$ be a real Banach space. We will denote by $B_{X}[x, r]$ the closed ball of radius $r>0$ around $x \in X$. We will identify any element $x \in X$ with its canonical image in $X^{* *}$. Unless otherwise specified, all subspaces we consider are norm closed. Our notations are otherwise standard. Any unexplained terminology can be found in either 4] or 9].

Recall that a subspace $Y$ of $X$ is called 1-complemented or constrained if there is a norm 1 projection on $X$ with range $Y$.

Definition $1.1([7)$. A Banach space $X$ is said to have the finite-infinite intersection property $\left(I P_{f, \infty}\right)$ if every family of closed balls in $X$ with empty intersection contains a finite subfamily with empty intersection.

It is well known that dual spaces and their constrained subspaces have $I P_{f, \infty}$. By $w^{*}$-compactness of the dual ball and the Principle of Local Reflexivity, it can be shown (see e.g., [7]) that $X$ has the $I P_{f, \infty}$ if and only if any family of closed balls centred at points of $X$ that intersects in $X^{* *}$ also intersects in $X$. With this in mind, we define

Definition 1.2 (1]). A subspace $Y$ of $X$ is said to be an almost constrained $(A C)$ subspace of $X$ if any family of closed balls centred at points of $Y$ that intersects in $X$ also intersects in $Y$.

Thus, $X$ has the $I P_{f, \infty}$ if and only if $X$ is an $A C$-subspace of $X^{* *}$. Clearly, any constrained subspace is an $A C$-subspace. In the case of $I P_{f, \infty}$, whether the converse is also true remains an open question (see [12, Remark 2, page 60], also [6, $\mathrm{X}(10)])$. However, we will give an example to show that an $A C$-subspace need not, in general, be constrained.

Received by the editors August 9, 2002.

2000 Mathematics Subject Classification. Primary 46B20.

Key words and phrases. Finite-infinite intersection property $\left(I P_{f, \infty}\right)$, almost constrained $(A C)$ subspace, (weakly) Hahn-Banach smooth, (weakly) $U$-subspace.

This work was partially supported by IFCPAR grant no. 2601-1. 
In addition, we apply some tools and techniques developed in [1] to obtain sufficient conditions for an $A C$-subspace to be constrained, much in the spirit of [6, 7]. Our condition is in terms of functionals with "locally unique" Hahn-Banach (i.e., norm-preserving) extensions, which improves significantly upon all existing conditions of similar type, as noted in [3, 8, and has significantly simpler proof. As in [6, 7], these conditions actually imply the existence of a unique norm 1 projection.

Definition 1.3. Let $Y$ be subspace of $X$.

(a) For $y^{*} \in Y^{*}, \operatorname{HB}\left(y^{*}\right)=\left\{x^{*} \in X^{*}:\left.x^{*}\right|_{Y}=y^{*}\right.$ and $\left.\left\|x^{*}\right\|=\left\|y^{*}\right\|\right\}$.

(b) $Y$ is a $U$-subspace of $X$ if for any $y^{*} \in Y^{*}, \operatorname{HB}\left(y^{*}\right)$ is a singleton. $X$ is said to be Hahn-Banach smooth if $X$ is a $U$-subspace of $X^{* *}$.

(c) The duality mapping $D$ for $X$ is the set-valued map from $S(X)$ to $S\left(X^{*}\right)$ defined by

$$
D(x)=\left\{x^{*} \in S\left(X^{*}\right): x^{*}(x)=1\right\}, \quad x \in S(X) .
$$

(d) $x \in S(X)$ is a smooth point of $B(X)$ if $D(x)$ is a singleton.

(e) $Y$ is a weakly $U$-subspace of $X$ if for every $y^{*} \in D(S(Y)), \operatorname{HB}\left(y^{*}\right)$ is a singleton.

$X$ is weakly Hahn-Banach smooth if $X$ is a weakly $U$-subspace of $X^{* *}$.

If $Y$ is a $U$-subspace, or even a weakly $U$-subspace of $X$, then it satisfies our sufficient condition. It is shown in [8 Theorem 2] that an $A C$-subspace $Y$ is constrained in $X$ if every point of $S(Y)$ is a smooth point of $B(X)$. We show that this happens if and only if every subspace $Z$ of $Y$ is a weakly $U$-subspace of $X$. Thus, our condition is weaker.

It follows from our result that $X$ is smooth if and only if every subspace of $X$ is a weakly $U$-subspace. This parallels the classical result of Taylor-Foguel [15, 5] that $X^{*}$ is strictly convex if and only if every subspace of $X$ is a $U$-subspace.

\section{Some Characterizations AND A COUNTEREXAMPle}

We will use the following notation:

Notation. Let $Y$ be a subspace of $X$. For all $x \in X$,

$$
\mathfrak{P}(x)=\bigcap_{y \in Y} B_{Y}[y,\|x-y\|] .
$$

Clearly, $\mathfrak{P}(y)=\{y\}$ for all $y \in Y$. Also, $Y$ is an $A C$-subspace of $X$ if and only if $\mathfrak{P}(x) \neq \emptyset$ for all $x \in X$.

We recall a definition from [1].

Definition 2.1. Let $Y$ be a subspace of $X$. We define

$$
O(Y, X)=\{x \in X:\|x-y\| \geq\|y\| \text { for all } y \in Y\} .
$$

$O\left(X, X^{* *}\right)$ is denoted by $O(X)$.

The following proposition characterizes $A C$-subspaces.

Proposition 2.2. For a subspace $Y$ of $X$, the following are equivalent:

(a) $Y$ is an $A C$-subspace of $X$.

(b) For all $x \in X$, there exists $y \in Y$ and $z \in O(Y, X)$ such that $x=y+z$.

(c) For every subspace $Z$ of $X$ such that $Y \subseteq Z$ and $\operatorname{dim}(Z / Y)=1, Y$ is constrained in $Z$. 
Proof. (a) $\Rightarrow$ (b). Let $x_{0} \in X$. By (a), there exists $y_{0} \in \mathfrak{P}\left(x_{0}\right)$. This implies $\left\|y_{0}-y\right\| \leq\left\|x_{0}-y\right\|$ for all $y \in Y$. Or, putting $u=y_{0}-y,\|u\| \leq\left\|x_{0}-y_{0}+u\right\|$ for all $u \in Y$. That is, $z_{0}=x_{0}-y_{0} \in O(Y, X)$ and $x_{0}=y_{0}+z_{0}$.

(b) $\Rightarrow$ (c). Let $Z$ be as in (c). Then one can write $Z=\overline{\operatorname{span}}\left[Y \cup\left\{x_{0}\right\}\right]$ for some $x_{0} \in X$. By $(b)$, there exists $y_{0} \in Y$ and $z_{0} \in O(Y, X)$ such that $x_{0}=y_{0}+z_{0}$. It follows that $Z=Y \oplus \mathbb{R} z_{0}$. But then, by definition of $O(Y, X), \alpha z_{0}+y \mapsto y$ is a norm 1 projection from $Z$ onto $Y$.

(c) $\Rightarrow$ (a). By (c), for every $x \in X$, there is a norm 1 projection $P_{x}$ from $Z_{x}=\overline{\operatorname{span}}[Y \cup\{x\}]$ onto $Y$. Clearly, $P_{x}(x) \in \mathfrak{P}(x)$.

Recall that a hyperplane $H$ in $X$ is a subspace such that $H=\operatorname{ker}\left(x^{*}\right)$ for some $x^{*} \in S\left(X^{*}\right)$. Since $\operatorname{dim}(X / H)=1$, we get

Corollary 2.3. Suppose $H$ is a hyperplane in $X$. Then $H$ is an $A C$-subspace if and only if $H$ is constrained in $X$.

Corollary 2.4. A subspace $Y$ is an AC-subspace of $X$ if and only if there is a (not necessarily linear) map $P$ from $X$ onto $Y$ satisfying the following properties:

(a) $P^{2}=P$;

(b) $P(\lambda x)=\lambda P(x)$ for all $x \in X, \lambda \in \mathbb{R}$;

(c) $P(x+y)=P(x)+y$ for all $x \in X, y \in Y$;

(d) $\|P(x)\| \leq\|x\|$ for all $x \in X$.

Proof. If $P$ is as above, then clearly for any $x \in X, P(x) \in \mathfrak{P}(x)$. Thus, $Y$ is an $A C$-subspace of $X$.

Conversely, let $Y$ be an $A C$-subspace of $X$. For $z \in O(Y, X)$, let $Y_{z}=Y \oplus \mathbb{R} z$ and $P_{z}$ be a norm 1 projection from $Y_{z}$ onto $Y$. Observe that for $z_{1}, z_{2} \in O(Y, X)$, either $Y_{z_{1}} \cap Y_{z_{2}}=Y$ or $Y_{z_{1}}=Y_{z_{2}}$. By Proposition 2.2(b), $\bigcup_{z \in O(Y, X)} Y_{z}=X$. Define $P: X \rightarrow Y$ by $P(x)=P_{z}(x)$, if $x \in Y_{z}$. Then $P$ is well-defined and satisfies all the listed properties.

Remark 2.5. Proposition 2.2(a) $\Leftrightarrow$ (c) for the case of $I P_{f, \infty}$ was noted in [12, Theorem 5.9]. Corollary 2.3 was also noted in [1]. Corollary 2.4 for the case of $I P_{f, \infty}$ was noted in [8, Theorem 2]. In all these cases, our proof is simpler.

Let us note that in Proposition 2.2(b), the representation $x=y+z$ with $y \in Y$ and $z \in O(Y, X)$ need not be unique.

Example 2.6. We now give an example to show that an $A C$-subspace need not, in general, be constrained. We need the following result (we thank Professor T.S.S.R.K. Rao of ISI, Bangalore, for drawing our attention to this result).

Theorem 2.7 ([1]). There exist Banach spaces $Z \supseteq X$ with $\operatorname{dim}(Z / X)=2$ satisfying

(i) There is no projection with norm 1 from $Z$ onto $X$.

(ii) For every $\varepsilon>0$, there is a projection with norm $\leq 1+\varepsilon$ from $Z$ onto $X$.

(iii) For every $Y$ with $Z \supseteq Y \supseteq X$ and $\operatorname{dim}(Y / X)=1$, there is a projection with norm 1 from $Y$ onto $X$.

By Proposition 2.2. (iii) implies that $X$ is an $A C$-subspace of $Z$, while by (i), there is no norm 1 projection from $Z$ onto $X$.

Definition 2.8. (a) [10] A Banach space $X$ such that $X^{*}$ is isometrically isomorphic to $L^{1}(\mu)$ for some positive measure $\mu$ is called an $L^{1}$-predual.

(b) A Banach space is a $\mathcal{P}_{1}$-space if it is constrained in every superspace. 
Remark 2.9. (a) From the results of [10, Chapter 3], it follows that $X$ is a real $L^{1}$-predual with $I P_{f, \infty}$ if and only if $X$ is a real $\mathcal{P}_{1}$-space. In particular, $X$ is constrained in $X^{* *}$.

(b) It can be shown that the space $X$ in Example 2.6 is not constrained in $X^{* *}$. Therefore, it could have been a possible counterexample to the $I P_{f, \infty}$ question as well. But, from the construction in [11, it is clear that the space $X$ is a real $L^{1}$-predual, but not a real $\mathcal{P}_{1}$-space. Thus it lacks the $I P_{f, \infty}$.

\section{Some Sufficient COnditions}

We now obtain sufficient conditions for an $A C$-subspace to be constrained. Some preliminaries first. As in [1, we introduce the following notation.

Definition 3.1. Let $Y$ be a subspace of $X$. For $x \in X$ and $y^{*} \in Y^{*}$, put

$$
\begin{aligned}
U\left(x, y^{*}\right) & =\inf \left\{y^{*}(y)+\|x-y\|: y \in Y\right\}, \\
L\left(x, y^{*}\right) & =\sup \left\{y^{*}(y)-\|x-y\|: y \in Y\right\} .
\end{aligned}
$$

For $x^{*} \in X^{*}$, we will write $U\left(x, x^{*}\right)$ for $U\left(x,\left.x^{*}\right|_{Y}\right)$. Let $C(x)=\left\{x^{*} \in B\left(X^{*}\right)\right.$ : $\left.U\left(x, x^{*}\right)=L\left(x, x^{*}\right)\right\}$, for $x \in X$, and $C=\bigcap_{x \in X} C(x)$.

The following result is immediate from the proof of the Hahn-Banach Theorem (see, e.g., [14, Section 48]).

Lemma 3.2. Let $Y$ be a subspace of $X, x_{0} \notin Y$ and $y^{*} \in S\left(Y^{*}\right)$. Then $L\left(x_{0}, y^{*}\right) \leq$ $U\left(x_{0}, y^{*}\right)$ and $\alpha$ lies between these two numbers if and only if there exists $x^{*} \in$ $H B\left(y^{*}\right)$ with $x^{*}\left(x_{0}\right)=\alpha$.

Remark 3.3. It is clear that for any $x^{*} \in B\left(X^{*}\right)$ and $x \in X, L\left(x, x^{*}\right) \leq x^{*}(x) \leq$ $U\left(x, x^{*}\right)$ and for any $y^{*} \in S\left(Y^{*}\right), \operatorname{HB}\left(y^{*}\right)$ is singleton if and only if for all $x \in X$, $L\left(x, y^{*}\right)=U\left(x, y^{*}\right)$.

The next three results are from 11. We include the proofs for the sake of completeness.

Lemma 3.4. Let $Y$ be a subspace of $X$. For $x_{1}, x_{2} \in X$, the following are equivalent:

(a) $x_{2} \in \bigcap_{y \in Y} B_{X}\left[y,\left\|x_{1}-y\right\|\right]$.

(b) For all $x^{*} \in B\left(X^{*}\right), U\left(x_{2}, x^{*}\right) \leq U\left(x_{1}, x^{*}\right)$.

Proof. Clearly, $x_{2} \in \bigcap_{y \in Y} B_{X}\left[y,\left\|x_{1}-y\right\|\right]$ if and only if $\left\|x_{2}-y\right\| \leq\left\|x_{1}-y\right\|$, for all $y \in Y$.

(a) $\Rightarrow$ (b). If for all $y \in Y,\left\|x_{2}-y\right\| \leq\left\|x_{1}-y\right\|$, then for all $x^{*} \in B\left(X^{*}\right)$, $x^{*}(y)+\left\|x_{2}-y\right\| \leq x^{*}(y)+\left\|x_{1}-y\right\|$. Therefore, $U\left(x_{2}, x^{*}\right) \leq U\left(x_{1}, x^{*}\right)$.

(b) $\Rightarrow$ (a). Suppose $\left\|x_{2}-y_{0}\right\|>\left\|x_{1}-y_{0}\right\|$ for some $y_{0} \in Y$. Then there exists $\varepsilon>0$ such that $\left\|x_{2}-y_{0}\right\|-\varepsilon \geq\left\|x_{1}-y_{0}\right\|$. Choose $x^{*} \in B\left(X^{*}\right)$ such that $\left\|x_{1}-y_{0}\right\| \leq\left\|x_{2}-y_{0}\right\|-\varepsilon<x^{*}\left(x_{2}-y_{0}\right)-\varepsilon / 2$. Thus $U\left(x_{1}, x^{*}\right) \leq x^{*}\left(y_{0}\right)+\left\|x_{1}-y_{0}\right\|<$ $x^{*}\left(x_{2}\right)-\varepsilon / 2<U\left(x_{2}, x^{*}\right)$.

Proposition 3.5. Let $Y$ be a subspace of $X, x^{*} \in B\left(X^{*}\right)$ and $x_{0} \in X \backslash Y$. The following are equivalent:

(a) $x^{*} \in C\left(x_{0}\right)$.

(b) $\left\|\left.x^{*}\right|_{Y}\right\|=1$ and every $x_{1}^{*} \in H B\left(\left.x^{*}\right|_{Y}\right)$ takes the same value at $x_{0}$. 
(c) $\left\|\left.x^{*}\right|_{Y}\right\|=1$ and if $\left\{x_{\alpha}^{*}\right\} \subseteq S\left(X^{*}\right)$ is a net such that $\left.\left.x_{\alpha}^{*}\right|_{Y} \rightarrow x^{*}\right|_{Y}$ in the $w^{*}$-topology of $Y^{*}$, then $\lim _{\alpha} x_{\alpha}^{*}\left(x_{0}\right)=x^{*}\left(x_{0}\right)$.

(d) $\left\|\left.x^{*}\right|_{Y}\right\|=1$ and if $\left\{x_{n}^{*}\right\} \subseteq S\left(X^{*}\right)$ is a sequence such that $\left.\left.x_{n}^{*}\right|_{Y} \rightarrow x^{*}\right|_{Y}$ in the $w^{*}$-topology of $Y^{*}$, then $\lim x_{n}^{*}\left(x_{0}\right)=x^{*}\left(x_{0}\right)$.

Proof. (a) $\Leftrightarrow$ (b). Let $\left\|\left.x^{*}\right|_{Y}\right\|=\alpha$. Then $\alpha \leq\left\|x^{*}\right\| \leq 1$ and it suffices to show that $\alpha=1$. Let $x_{1}^{*} \in \operatorname{HB}\left(\left.x^{*}\right|_{Y}\right)$. Then $\left\|x_{1}^{*}\right\|=\alpha$ and therefore, for any $y \in Y$, $\left|x_{1}^{*}\left(x_{0}-y\right)\right| \leq \alpha\left\|x_{0}-y\right\| \leq\left\|x_{0}-y\right\|$. It follows that

$$
\begin{aligned}
L\left(x_{0}, x^{*}\right) & \leq \sup \left\{x^{*}(y)-\alpha\left\|x_{0}-y\right\|: y \in Y\right\} \leq x_{1}^{*}\left(x_{0}\right) \\
& \leq \inf \left\{x^{*}(y)+\alpha\left\|x_{0}-y\right\|: y \in Y\right\} \leq U\left(x_{0}, x^{*}\right) .
\end{aligned}
$$

Since $x^{*} \in C\left(x_{0}\right)$, equality holds everywhere.

Now if $\alpha<1$, let $0<\delta<d\left(x_{0}, Y\right)$ and let $0<\varepsilon<(1-\alpha) \delta$. Then for all $y \in Y$, $(1-\alpha)\left\|x_{0}-y\right\|>\varepsilon$. Therefore, for all $y \in Y$,

$$
y^{*}(y)-\left\|x_{0}-y\right\|+\varepsilon<y^{*}(y)-\alpha\left\|x_{0}-y\right\| .
$$

Thus, the first inequality must be strict. Contradiction!

The result now follows from Lemma 3.2 .

(b) $\Rightarrow(\mathrm{c})$. Let $\left\{x_{\alpha}^{*}\right\} \subseteq S\left(X^{*}\right)$ be a net such that $\lim _{\alpha} x_{\alpha}^{*}(y)=x^{*}(y)$ for all $y \in Y$. It follows that any $\mathrm{w}^{*}$-cluster point of $\left\{x_{\alpha}^{*}\right\}$ is in $\operatorname{HB}\left(\left.x^{*}\right|_{Y}\right)$. By (b), therefore, $\lim x_{\alpha}^{*}\left(x_{0}\right)=x^{*}\left(x_{0}\right)$.

(c) $\Rightarrow(\mathrm{d})$ is clear.

(d) $\Rightarrow$ (b). If $x_{1}^{*} \in \operatorname{HB}\left(\left.x^{*}\right|_{Y}\right)$ with $x^{*}\left(x_{0}\right) \neq x_{1}^{*}\left(x_{0}\right)$, then the constant sequence $x_{n}^{*}=x_{1}^{*}$ clearly satisfies $\lim _{n} x_{n}^{*}(y)=x^{*}(y)$ for all $y \in Y$, but $\left\{x_{n}^{*}\left(x_{0}\right)\right\}$ cannot converge to $x^{*}\left(x_{0}\right)$.

Proposition 3.6. Let $Y$ be a subspace of $X$. For $x^{*} \in B\left(X^{*}\right)$, the following are equivalent:

(a) $x^{*} \in C$.

(b) $\left\|\left.x^{*}\right|_{Y}\right\|=1$ and $H B\left(\left.x^{*}\right|_{Y}\right)=\left\{x^{*}\right\}$.

(c) $\left\|\left.x^{*}\right|_{Y}\right\|=1$ and if $\left\{x_{\alpha}^{*}\right\} \subseteq S\left(X^{*}\right)$ is a net such that $\left.\left.x_{\alpha}^{*}\right|_{Y} \rightarrow x^{*}\right|_{Y}$ in the $w^{*}$-topology of $Y^{*}$, then $x_{\alpha}^{*} \rightarrow x^{*}$ in the $w^{*}$-topology of $X^{*}$.

(d) $\left\|\left.x^{*}\right|_{Y}\right\|=1$ and if $\left\{x_{n}^{*}\right\} \subseteq S\left(X^{*}\right)$ is such that $\left.\left.x_{n}^{*}\right|_{Y} \rightarrow x^{*}\right|_{Y}$ in the $w^{*}$ topology of $Y^{*}$, then $x_{n}^{*} \rightarrow x^{*}$ in the $w^{*}$-topology of $X^{*}$.

Here is our first sufficient condition for an $A C$-subspace to be constrained.

Proposition 3.7. For a subspace $Y$ of $X$, the following are equivalent:

(a) $Y$ is an $A C$-subspace of $X$ and $O(Y, X)$ is a closed subspace of $X$.

(c) $Y$ is an $A C$-subspace of $X$ and $O(Y, X)$ is a linear subspace of $X$.

(c) $Y$ is constrained in $X$ and for all $x \in X, \mathfrak{P}(x)$ is a singleton.

Moreover, in this case, $Y$ is constrained by a unique norm 1 projection.

Proof. (a) $\Rightarrow$ (b) is trivial.

(b) $\Rightarrow$ (c). Since $Y$ is an $A C$-subspace of $X$, by Proposition 2.2 any $x \in X$ can be written as $x=y+z$, where $y \in Y$ and $z \in O(Y, X)$. Since both $Y$ and $O(Y, X)$ are linear subspaces and $Y \cap O(Y, X)=\{0\}$, this representation is unique and $x \mapsto y$ is a well-defined linear map. Since $z \in O(Y, X)$, this map is of norm 1 . Hence $Y$ is constrained in $X$. Moreover, since $y \in \mathfrak{P}(x), \mathfrak{P}(x)$ is single-valued.

(c) $\Rightarrow$ (a). Let $Y$ be constrained in $X$ by a norm 1 projection $P$ and for all $x \in X$, let $\mathfrak{P}(x)$ be a singleton. Clearly, $Y$ is an $A C$-subspace of $X$ and for all 
$x \in X, \mathfrak{P}(x)=\{P(x)\}$. It is easy to see that $\operatorname{ker}(P) \subseteq O(Y, X)$ and since for all $x \in X, \mathfrak{P}(x)=\{P(x)\}, \operatorname{ker}(P) \supseteq O(Y, X)$. Thus, $O(Y, X)=\operatorname{ker}(P)$ is a closed subspace of $X$.

Remark 3.8. (a) Even in the case of $I P_{f, \infty}$, this observation is new. References [6] and [7] discuss more complicated situations when $O(X)$, being a linear subspace of $X^{* *}$, automatically implies that it is a $\mathrm{w}^{*}$-closed subspace of $X^{* *}$.

(b) We do not know if (c) can be replaced by " $Y$ is constrained by a unique norm 1 projection".

(c) It follows from the proof that

$$
\bigcup\{\operatorname{ker}(P): P \text { is a norm } 1 \text { projection onto } Y\} \subseteq O(Y, X) .
$$

Are these two sets equal?

The following result significantly improves [3, Lemma 2], which was also the key tool in 8 .

Lemma 3.9. Let $Y$ be a subspace of $X$. Let $x_{1}, x_{2} \in X$ be such that $x_{1} \in$ $\bigcap_{y \in Y} B_{X}\left[y,\left\|x_{2}-y\right\|\right]$. Then for any $x^{*} \in C\left(x_{2}\right), x^{*}\left(x_{1}-x_{2}\right)=0$.

Proof. Let $x_{1}, x_{2} \in X$ be such that $x_{1} \in \bigcap_{y \in Y} B_{X}\left[y,\left\|x_{2}-y\right\|\right]$. Then, by Lemma 3.4, for all $x^{*} \in B\left(X^{*}\right)$,

$$
L\left(x_{2}, x^{*}\right) \leq L\left(x_{1}, x^{*}\right) \leq U\left(x_{1}, x^{*}\right) \leq U\left(x_{2}, x^{*}\right) .
$$

Thus for $x^{*} \in C\left(x_{2}\right)$, equality holds. By Lemma 3.2, the result follows.

Here is our main theorem.

Theorem 3.10. Let $Y$ be a subspace of $X$. Suppose

$$
\text { for every } x_{1}, x_{2} \in X, \quad C\left(x_{1}\right) \cap C\left(x_{2}\right) \text { separates points of } Y .
$$

If $Y$ is an AC-subspace of $X$, then $Y$ is constrained in $X$. Moreover, the projection is unique and $O(Y, X)$ is a closed subspace of $X$.

Proof. Since $Y$ is an $A C$-subspace of $X, \mathfrak{P}(x) \neq \emptyset$ for all $x \in X$. By Lemma 3.9 for all $x \in X$,

$$
x^{*}(x-y)=0 \quad \text { for any } x^{*} \in C(x), y \in \mathfrak{P}(x) .
$$

Now if $y_{1}, y_{2} \in \mathfrak{P}(x)$, then for any $x^{*} \in C(x), x^{*}\left(x-y_{1}\right)=x^{*}\left(x-y_{2}\right)=0$. Therefore, $x^{*}\left(y_{1}-y_{2}\right)=0$. By (1), $y_{1}=y_{2}$. That is, $\mathfrak{P}(x)$ is single-valued. Let $\mathfrak{P}(x)=\{P(x)\}$. Then, $P$ satisfies all the properties listed in Corollary 2.4 So, it only remains to show that $P$ is additive.

Let $x_{1}, x_{2} \in X$. If $x^{*} \in C\left(x_{1}\right) \cap C\left(x_{2}\right)$, then by Proposition 3.5, $x^{*} \in C\left(x_{1}+x_{2}\right)$ and by (2), $x^{*}\left(x_{1}-P\left(x_{1}\right)\right)=x^{*}\left(x_{2}-P\left(x_{2}\right)\right)=x^{*}\left(\left(x_{1}+x_{2}\right)-P\left(x_{1}+x_{2}\right)\right)=0$. Therefore, $x^{*}\left(P\left(x_{1}+x_{2}\right)-P\left(x_{1}\right)-P\left(x_{2}\right)\right)=0$. By (1), $P\left(x_{1}+x_{2}\right)=P\left(x_{1}\right)+P\left(x_{2}\right)$.

The rest of the result follows from Proposition 3.7.

By Theorem [3.10 the condition " $C$ separates points of $Y$ " is sufficient for an $A C$-subspace to be constrained by a unique norm 1 projection. This condition is clearly satisfied if $Y$ is a $U$-subspace, or even a weakly $U$ subspace of $X$.

It is shown in [8, Theorem 2] that an $A C$-subspace $Y$ is constrained in $X$ by a unique norm 1 projection if every point of $S(Y)$ is a smooth point of $B(X)$. By the following result, our condition is much weaker. 
Proposition 3.11. Every point of $S(Y)$ is a smooth point of $B(X)$ if and only if every subspace $Z$ of $Y$ is a weakly $U$-subspace of $X$. In particular, $X$ is smooth if and only if every subspace of $X$ is a weakly $U$-subspace of $X$.

Proof. Suppose every point of $S(Y)$ is a smooth point of $B(X)$. Let $Z$ be any subspace of $Y$. Suppose $z^{*} \in S\left(Z^{*}\right)$ attains its norm at $z_{0} \in S(Z)$. By assumption, $z_{0}$ is a smooth point of $B(X)$. Now, $z^{*} \in D_{Z}\left(z_{0}\right)$ and $\operatorname{HB}\left(z^{*}\right) \in D_{X}\left(z_{0}\right)$. Since $D_{X}\left(z_{0}\right)$ is a singleton, so is $\operatorname{HB}\left(z^{*}\right)$. Thus, $Z$ is a weakly $U$-subspace of $X$.

Conversely, suppose there exists $y_{0} \in S(Y)$ such that $D_{X}\left(y_{0}\right)$ is not a singleton. Suppose $\left\{x_{1}^{*}, x_{2}^{*}\right\} \subseteq D_{X}\left(y_{0}\right)$ and $x_{1}^{*} \neq x_{2}^{*}$. Let $Z=\left\{x \in Y: x_{1}^{*}(x)=x_{2}^{*}(x)\right\}$. Then $y_{0} \in S(Z)$ and therefore, $\left\|\left.x_{1}^{*}\right|_{Z}\right\|=\left\|\left.x_{2}^{*}\right|_{Z}\right\|=1$. Thus, $z^{*}=\left.x_{1}^{*}\right|_{Z} \in S\left(Z^{*}\right)$ attains its norm at $y_{0} \in S(Z)$, but $\left\{x_{1}^{*}, x_{2}^{*}\right\} \subseteq \operatorname{HB}\left(z^{*}\right)$.

Example 3.12. As noted in [6], the space $X=L^{\infty}$ gives an example of a dual space such that there are infinitely many norm 1 projections from $X^{* *}$ onto $X$. This produces an example of a space with $I P_{f, \infty}$ that is constrained in $X^{* *}$, but $O(X)$ is not a closed subspace of $X^{* *}$. This also shows that our sufficient condition, although weaker than the known ones, is still not necessary for an $A C$-subspace to be constrained.

We conclude the paper with some necessary and/or sufficient conditions for $O(Y, X)$ to be a closed subspace of $X$. First we need a characterization of $O(Y, X)$. This is a slight improvement over that in [1].

Definition 3.13. We say $A \subseteq B\left(X^{*}\right)$ is a norming set for $X$ if $\|x\|=\sup \left\{x^{*}(x)\right.$ : $\left.x^{*} \in A\right\}$ for all $x \in X$.

A subspace $F$ of $X^{*}$ is called a norming subspace if $B(F)$ is a norming set for $X$.

Lemma 3.14. Let $Y$ be a subspace of $X$. For $x \in X$, the following are equivalent:

(a) $x \in O(Y, X)$.

(b) $\left.\operatorname{ker}(x)\right|_{Y} \subseteq Y^{*}$ is a norming subspace for $Y$.

(c) $0 \in \bigcap_{y \in Y} B_{Y}[y,\|x-y\|]$.

(d) For every $x^{*} \in B\left(X^{*}\right), L\left(x, x^{*}\right) \leq 0 \leq U\left(x, x^{*}\right)$.

(e) For every $y^{*} \in B\left(Y^{*}\right), L\left(x, y^{*}\right) \leq 0 \leq U\left(x, y^{*}\right)$.

Further, for a $w^{*}$-closed subspace $F \subseteq X^{*},\left.F\right|_{Y}$ is a norming subspace for $Y$ if and only if $F_{\perp} \subseteq O(Y, X)$, where $F_{\perp}=\{x \in X: f(x)=0$ for all $f \in F\}$.

Proof. Let $F \subseteq X^{*}$ be a $w^{*}$-closed subspace such that $F_{\perp} \subseteq O(Y, X)$. Then $F=\left(X / F_{\perp}\right)^{*}$ and therefore, it suffices to show that $\|y\|=\left\|y+F_{\perp}\right\|=d\left(y, F_{\perp}\right)$.

Clearly, $\|y\| \geq d\left(y, F_{\perp}\right)$. Also, since $F_{\perp} \subseteq O(Y, X)$, for any $y \in Y$ and $z \in F_{\perp}$, $\|y+z\| \geq\|y\|$. Thus, $d\left(y, F_{\perp}\right) \geq\|y\|$.

Specializing to $F=\operatorname{ker}(x)$, we get $(\mathrm{a}) \Rightarrow(\mathrm{b})$.

(b) $\Rightarrow$ (a). Since $\left.\operatorname{ker}(x)\right|_{Y}$ norms $Y,\|y\|=\left\|\left.y\right|_{\operatorname{ker}(x)}\right\|=d(y, \mathbb{R} x)$ for all $y \in Y$. Hence $\|x-y\| \geq \inf _{\lambda \in \mathbb{R}}\|y-\lambda x\|=\|y\|$ for all $y \in Y$. Thus, $x \in O(Y, X)$.

Now suppose $F \subseteq X^{*}$ is a w $^{*}$-closed subspace such that $\left.F\right|_{Y}$ is a norming subspace for $Y$. If $x \in F_{\perp}$, then $F \subseteq \operatorname{ker}(x)$ and therefore, $x \in O(Y, X)$. That is, $F_{\perp} \subseteq O(Y, X)$.

(a) $\Leftrightarrow$ (c) and (d) $\Rightarrow$ (e) are immediate from definition, while (c) $\Rightarrow$ (d) follows from Lemma 3.4. 
(e) $\Rightarrow$ (a). For every $y^{*} \in B\left(Y^{*}\right), 0 \leq U\left(x, y^{*}\right)$ implies for all $y^{*} \in B\left(Y^{*}\right)$ and $y \in Y$,

$$
0 \leq y^{*}(y)+\|x-y\| \Longrightarrow y^{*}(-y) \leq\|x-y\| .
$$

Since this is true for all $y^{*} \in B\left(Y^{*}\right),\|y\| \leq\|x-y\|$ for all $y \in Y$. That is, $x \in O(Y, X)$.

Let $\mathcal{N}=\left\{F: F\right.$ is a $\mathrm{w}^{*}$-closed subspace of $X^{*}$ and $\left.F\right|_{Y}$ is a norming subspace for $Y$ \} and $N=\bigcap \mathcal{N}$. Similar to [6], we observe

Proposition 3.15. Let $Y$ be a subspace of $X . O(Y, X)$ is a closed subspace of $X$ if and only if $\left.N\right|_{Y}$ is a norming subspace for $Y$. In particular, this happens if $\left.C\right|_{Y}$ is a norming set for $Y$.

Proof. By Lemma 3.14 $F \in \mathcal{N}$ if and only if $F_{\perp} \subseteq O(Y, X)$. Thus if $\left.N\right|_{Y}$ norms $Y$, then $N \in \mathcal{N}$ and hence, $N_{\perp} \subseteq O(Y, X)$. On the other hand, if $x \in O(Y, X)$, then $\operatorname{ker}(x) \in \mathcal{N}$, and hence, $N \subseteq \operatorname{ker}(x)$. That is, $x \in N_{\perp}$. Therefore, $O(Y, X)=N_{\perp}$, and $O(Y, X)$ is a closed subspace of $X$.

Conversely, if $O(Y, X)$ is a closed subspace of $X$ and $M=O(Y, X)^{\perp}$, then $M_{\perp}=$ $O(Y, X)$ and therefore, $M \in \mathcal{N}$. Moreover, for every $F \in \mathcal{N}, F_{\perp} \subseteq O(Y, X)=M_{\perp}$, and hence, $M \subseteq F$. This shows $N=M$ and $N \in \mathcal{N}$.

Now, if $\left.C\right|_{Y}$ is a norming set for $Y$, then as above, $C_{\perp} \subseteq O(Y, X)$.

Conversely let $x \in O(Y, X)$. Let $x^{*} \in C$. By Lemmas 3.2 and 3.14, there exists $z^{*} \in \operatorname{HB}\left(\left.x^{*}\right|_{Y}\right)$ such that $z^{*}(x)=0$. Since $x^{*} \in C, \operatorname{HB}\left(\left.x^{*}\right|_{Y}\right)=\left\{x^{*}\right\}$, and we have $x^{*}(x)=0$. Thus, $C_{\perp}=O(Y, X)$.

Definition 3.16. (a) [16] Let $Y$ be a subspace of $X$. Let

$$
A(Y)=\left\{x^{*} \in B\left(X^{*}\right):\left.x^{*}\right|_{Y} \text { is an extreme point of } B\left(Y^{*}\right)\right\} \text {. }
$$

$Y$ is a weakly separating subspace of $X$ if $Y$ separates points of $A(Y)$.

(b) 9 A subspace $Y \subseteq X$ is said to be an $M$-ideal if there exists a subspace $N \subseteq X^{*}$ such that $X^{*}=Y^{\perp} \oplus_{1} N$.

Proposition 3.17. In each of the following cases, $O(Y, X)$ is a closed subspace of $X$, a fortiori, if $Y$ is an $A C$-subspace, then $Y$ is constrained by a unique norm 1 projection.

(a) $Y$ is a weakly separating subspace of $X$.

(b) $Y$ is an $M$-ideal in $X$.

(c) $Y$ is a subspace of $X=C(K)$ containing the constants and separating points of $K$.

Proof. (a) A careful examination of the proof of [16, Lemma 1] actually shows that $A(Y) \subseteq C$. It is easy to see that $A(Y)$ is a norming set for $Y$. The result follows from Proposition 3.15

(b) [9, Theorem I.1.12] observes that an $M$-ideal is a $U$-subspace.

(c) As observed in [16], such a $Y$ is weakly separating.

Remark 3.18. (a) In [16], it is shown that for a weakly separating subspace in $C(K)$, if there is a norm 1 projection, it must be unique. Clearly, our conclusion is stronger.

(b) In [13], it is shown that an $M$-ideal with the $I P_{f, \infty}$ is an $M$-summand. An argument similar to [2, Proposition 2.8] shows that an $M$-ideal $Y$ in $X$ with the 
$I P_{f, \infty}$ is an $A C$-subspace of $X$. Thus, Proposition 3.17 (b) improves the result in [13].

\section{ACKNOWLEDGEMENTS}

Part of this work was done during the first-named author's visit to the Southern Illinois University at Edwardsville, USA, in May-June 2002. He would like to thank Professor K. Jarosz for the warm hospitality and many fruitful discussions. We also thank the referee for some suggestions that improved the readability of the paper.

\section{REFERENCES}

[1] P. Bandyopadhayay, S. Basu, S. Dutta and B. L. Lin, Very nonconstrained subspaces of Banach spaces, Preprint 2002 (submitted).

[2] Pradipta Bandyopadhyay and T. S. S. R. K. Rao, Central subspaces of Banach spaces, J. Approx. Theory, 103 (2000), 206-222. MR 2001b:46022

[3] B. Beuzamy and B. Maurey, Points minimaux et ensembles optimaux dans les espaces de Banach, J. Functional Analysis, 24 (1977), 107-139. MR 55:1044

[4] J. Diestel and J. J. Uhl, Jr., Vector measures, Mathematical Surveys, No. 15, Amer. Math. Soc., Providence, R. I. (1977). MR 56:12216

[5] S. R. Foguel, On a Theorem by A. E. Taylor, Proc. Amer. Math. Soc., 9 (1958), 325. MR 20:219

[6] G. Godefroy, Existence and uniqueness of isometric preduals: a survey, Banach space theory (Iowa City, IA, 1987), 131-193, Contemp. Math., 85, Amer. Math. Soc., Providence, RI, 1989. MR 90b:46035

[7] G. Godefroy and N. J. Kalton, The ball topology and its applications, Banach space theory (Iowa City, IA, 1987), 195-237, Contemp. Math., 85, Amer. Math. Soc., Providence, RI, 1989. MR 90c: 46022

[8] G. Godini, On minimal points, Comment. Math. Univ. Carolina, 21 (1980), 407-419. MR 81j:46023

[9] P. Harmand, D. Werner and W. Werner, $M$-ideals in Banach spaces and Banach algebras, Lecture Notes in Mathematics, 1547, Springer-Verlag, Berlin, 1993. MR 94k:46022

[10] H. E. Lacey, Isometric theory of classical Banach spaces, Die Grundlehren der mathematischen Wissenschaften, Band 208, Springer-Verlag, New York-Heidelberg, 1974. MR 58:12308

[11] J. Lindenstrauss, On projections with norm 1-an example, Proc. Amer. Math. Soc., 15 (1964), 403-406. MR 28:4335

[12] J. Lindenstrauss, Extension of compact operators, Mem. Amer. Math. Soc., No. 48, 1964. MR 31:3828

[13] T. S. S. R. K. Rao, Intersection properties of balls in tensor products of some Banach spaces, II, Indian J. Pure Appl. Math., 21 (1990), 275-284. MR 91g:46020

[14] G. F. Simmons, Introduction to topology and modern analysis, McGraw-Hill Book Co., Inc., New York-San Francisco, Calif.-Toronto-London (1963). MR 26:4145

[15] A. E. Taylor, The extension of linear functionals, Duke Math. J., 5 (1939), 538-547. MR $1: 58 \mathrm{~b}$

[16] D. E. Wulbert, Some complemented function spaces in $C(X)$, Pacific J. Math., 24 (1968), 589-602. MR 36:6915

Statistics and Mathematics Division, Indian Statistical Institute, 203, B. T. Road, KolKata 700 108, INDIA

E-mail address: pradipta@isical.ac.in

Statistics and Mathematics Division, Indian Statistical Institute, 203, B. T. Road, KolKata 700 108, InDia

E-mail address: sudipta_r@isical.ac.in 